\title{
Evaluation of the physi- and chemisorption of hydrogen in alkali (Na,
}

\section{Li) doped fullerenes}

Patrick A. Ward ${ }^{\ddagger}, \ddagger$, Joseph A. Teprovich Jr., R. N. Compton ${ }^{\ddagger}$, Viviane Schwartz ${ }^{\dagger}$, Gabriel M. Veith $^{\dagger}$, Ragaiy Zidan ${ }^{\ddagger *}$

$¥$ Department of Chemistry, University of Tennessee Knoxville, Knoxville, TN 37996, USA.

‡ Clean Energy Directorate, Center for Hydrogen Research, Savannah River National Lab, Aiken, SC 28903, USA.

† Oak Ridge National Laboratory, Oak Ridge, TN 37831, USA.

\begin{abstract}
In this study, alkali doped fullerenes synthesized by two different solvent assisted mixing techniques are compared for their hydrogen uptake activity. In particular, we investigated the interaction of hydrogen with lithium and sodium doped fullerenes via physisorption. In addition, we present the first mass spectrometric evidence for the formation of $\mathrm{C}_{60} \mathrm{H}_{60}$ via chemisorption. Hydrogen physisorption isotherms up to $1 \mathrm{~atm}$ at temperatures ranging from $77 \mathrm{~K}$ to $303 \mathrm{~K}$ were measured demonstrating an increase in hydrogen uptake versus pure $\mathrm{C}_{60}$ and increased isosteric heats of adsorption for the lithium doped fullerene $\mathrm{Li}_{12} \mathrm{C}_{60}$. The hydrogen uptake in $\mathrm{Na}_{6} \mathrm{C}_{60}$, $\mathrm{Li}_{6} \mathrm{C}_{60}$, and $\mathrm{Li}_{12} \mathrm{C}_{60}$ was enhanced compared to pure $\mathrm{C}_{60}$. However, despite these improvements the low amount of physisorbed hydrogen at $1 \mathrm{~atm}$ and $77 \mathrm{~K}$ in these materials suggests that fullerenes do not possess enough accessible surface area to effectively store hydrogen due to their close packed crystalline nature.
\end{abstract}

Keywords: Hydrogen Storage, fullerenes, $\mathrm{C}_{60} \mathrm{H}_{60}$, physisorption, chemisorption.

*Corresponding Author Tel: (803) 646-8876 E-mail: Ragaiy.Zidan@srnl.doe.gov 


\section{Introduction}

Storing hydrogen safely and efficiently with renewable resources is of great interest for the development of clean energy technologies incorporating hydrogen as an energy carrier. The most common approaches for storing hydrogen in materials are via physisorption in porous materials, storage in metal hydrides, chemical storage, or Kubas bound hydrogen (weak covalent bond) in which the intermolecular binding strength is between that of physisorption and chemisorption. ${ }^{[1]}$ It has been proposed by various theoretical studies that alkali decorated fullerenes could be capable of storing a significant amount of hydrogen in a Kubas fashion on the alkali metals bound to the fullerene cage. ${ }^{[2-4]}$ Investigation of hydrogen storage materials based on carbon is further inspired by the abundance of carbon on Earth, the wide range of material properties that can be obtained through various allotropes of carbon, and the low atomic mass of carbon. Since high gravimetric densities of hydrogen are required for application in light duty PEM fuel cell vehicles, light elements are required for chemisorption based materials. It is well known that the gravimetric hydrogen densities of many hydrocarbons, such as octane $(15.8 \mathrm{wt} \%$ $\mathrm{H}_{2}$ ), are sufficient to satisfy DOE requirements. Furthermore, the volumetric hydrogen density in octane $\left(0.1109 \mathrm{~g} / \mathrm{mL}\right.$ or $0.055 \mathrm{~mol} \mathrm{H} / \mathrm{mL}$ at $\left.25^{\circ} \mathrm{C}\right)$ is higher than that of liquefied hydrogen (0.07 g/mL or $\left.0.0348 \mathrm{~mol} \mathrm{H}_{2} / \mathrm{mL}\right)$. Unfortunately, hydrogen storage applications for alkanes have been hindered by incomplete conversion to the alkene, low reversibility, and the requirement of precious metal catalysts. ${ }^{[5]}$ Therefore, the most logical carbon-based compounds for hydrogen storage would be materials, such as fullerenes, which are capable of complete reversibility without the need for precious metal catalysts. Previous work by our group has demonstrated enhancements in the hydrogen storage properties of complex metal hydrides using $\mathrm{C}_{60}$ and carbon nanotubes. ${ }^{[6-9]}$ It was determined that the addition of $\mathrm{C}_{60}$ to $\mathrm{LiBH}_{4}$ lowers the 
dehydrogenation temperature of $\mathrm{LiBH}_{4}$ and the pressure and temperature requirements for its regeneration. It was also determined that carbon nanomaterials can have a catalytic effect on the dehydrogenation of $\mathrm{NaAlH}_{4}$ by destabilizing the $\mathrm{AlH}_{4}$ anion. We have also previously investigated alkali doped fullerenes by anelastic spectroscopy as well as other spectroscopic methods and reported a comparison of the chemisorption properties of sodium and lithium doped fullerenes by the hydride doping method. ${ }^{[10-12]}$ These previous results show increased hydrogen uptake for alkali hydride doped fullerenes.

Direct hydrogenation of fullerenes to produce fulleranes (hydrogenated fullerenes) is well known. ${ }^{[13]}$ It has been demonstrated that fulleranes more reduced than $\mathrm{C}_{60} \mathrm{H}_{36}$ can be produced with extreme conditions $\left(\mathrm{T}=400{ }^{\circ} \mathrm{C}, \mathrm{P}=120\right.$ bar). While the mixture of fulleranes possessed an average of $\sim 31$ hydrogens per $\mathrm{C}_{60},{ }^{[14,15]}$ fulleranes with a hydrogen content greater than $\mathrm{C}_{60} \mathrm{H}_{36}$ were observed by mass spectrometry. It was further discovered that temperatures above $400{ }^{\circ} \mathrm{C}$ can result in cage fragmentation. Recently, we have shown that lithium doped fullerenes can enhance the reversibility and reduce the temperature and pressure required $\left(\mathrm{T}=350{ }^{\circ} \mathrm{C}, \mathrm{P}=105\right.$ bar) to produce highly reduced fullerenes up to $\mathrm{C}_{60} \mathrm{H}_{48}$ with an average of $\sim 40$ hydrogens per $\mathrm{C}_{60}$. ${ }^{[16]}$ Similar enhancements of the chemisorption of hydrogen in $\mathrm{Li}_{12} \mathrm{C}_{60}$ has also been demonstrated. ${ }^{[17]}$ It has also been previously reported by our group and Mauron et al that sodium doping of fullerenes leads to increased hydrogen capacities via chemisorption. ${ }^{[18,19]}$

Another avenue for hydrogen storage is via physisorption of hydrogen on alkali doped fullerenes. Density functional theory calculations suggest that lithium decorated fullerenes could store large amounts of hydrogen via physisorption with binding energies comparable to that of Kubas bound $\mathrm{H}_{2}{ }^{[4]}$ The high hydrogen uptake predicted is attributed to a charge transfer of electron density to the fullerene cage from the lithium atom. Based on the DFT calculations, it 
was predicted that the charge differential created would polarize the molecular orbital of hydrogen leading to an enhanced electrostatic attraction between the hydrogen molecule and lithium located on the surface of the fullerene cage. However, these theoretical studies were conducted on an isolated alkali doped fullerene and the actual solid state crystal lattice ensemble was not considered due to extensive computation requirements.

Herein, we report our efforts to experimentally evaluate the enhancement of hydrogen physisorption in alkali $(\mathrm{Na}, \mathrm{Li})$ doped $\mathrm{C}_{60}$. We also report a comparison of different solvent assisted mixing methods (in THF or $\mathrm{NH}_{3}$ ) and alkali metal source (hydride or metal). Additionally, we also examined the chemisorption of highly hydrogenated $\mathrm{Na}_{6} \mathrm{C}_{60}$ by LDI-TOFMS. Hydrogen and deuterium chemisorption studies on this material are consistent with the formation of $\mathrm{C}_{60} \mathrm{H}_{60}$.

\section{Experimental}

\subsection{Chemicals}

Anhydrous ammonia was purchased from Air Gas and was delivered to reaction vessels through a drying tube filled with Dryrite. Sodium hydride (95\%), lithium hydride (95\%, 30 mesh), Sodium (99\%) and Lithium (99\%)were purchased from Sigma Aldrich and used as received. $\mathrm{C}_{60}(99.5 \%)$ was purchased from SES corp. and was used without further purification.

\subsection{Synthesis}

Lithium and sodium doped fullerenes were prepared via two different methods, which will be referred to in this study as "hydride doped" and " $\mathrm{NH}_{3}$ mixed". Hydride doped sodium 
and lithium $\mathrm{C}_{60}$ were synthesized via solvent assisted mixing in THF, solvent removal, and annealing as described in previous work. ${ }^{[16,18]}$

Lithium and sodium doped fullerenes were also prepared by solvent assisted mixing in liquid ammonia. Lithium and sodium to $\mathrm{C}_{60}$ ratios $6: 1$ and 12:1 were prepared by adding the appropriate molar ratios of alkali metal and $\mathrm{C}_{60}(250-500 \mathrm{mg}$ scale) to a 3-neck round bottom flask equipped with a dry ice/acetone condenser and flow control stopcocks under an Ar atmosphere. The apparatus was then connected to a Schlenk line and purged with UHP Ar, followed by addition of approximately $150 \mathrm{~mL}$ of liquid ammonia by condensation. Upon solvation of the lithium metal a deep red color is observed as well as solvation of the $\mathrm{C}_{60}$. The reaction mixture was then stirred for 8-12 hours followed by solvent removal under Ar flow at $\sim 80{ }^{\circ} \mathrm{C}$. The product was then collected and annealed under vacuum at $200{ }^{\circ} \mathrm{C}$.

\subsection{Instrumental Techniques}

Laser desorption ionization time-of-flight mass spectrometry (LDI-TOF-MS) was carried out on an Applied Biosystems Voyager DE-Pro using delayed pulse extraction in reflectron mode with a $337.1 \mathrm{~nm}$ nitrogen laser. Positive mode spectra were recorded with a spectrum consisting of 50 averaged spectra. Typical mass resolution $(\mathrm{m} / \Delta \mathrm{m})$ was 2,500 and was calculated using the full width half maximum approach. Samples were prepared, under an argon atmosphere, by spotting the plate with $1 \mu \mathrm{L}$ of a $0.5 \mathrm{mg} / \mathrm{mL}$ solution of the sample in THF. The sample plate was contained in an argon filled container for transfer to the spectrometer, but 5-10 seconds of air exposure were required to insert the sample plate into the instrument. In order to determine if oxidation had occurred, the samples were left in air overnight after measurements to observe differences between the two mass spectra. It was noticed that peaks consistent with 
$\mathrm{C}_{60} \mathrm{H}_{\mathrm{x}} \mathrm{O}$ were noticed after overnight air exposure, but were not noticed in spectra with 5-10 seconds of air exposure.

The hydrogen chemisorption experiments were carried out on a HyEnergy PCT Pro 2000 (Sievert's Apparatus) with a sample size of $\sim 100 \mathrm{mg}$ with 100 bar of $\mathrm{H}_{2}$ overpressure. The temperature was ramped from $25^{\circ} \mathrm{C}$ to the target temperature $\left(300{ }^{\circ} \mathrm{C}\right.$ or $\left.350{ }^{\circ} \mathrm{C}\right)$ over 1.5 hours followed by isothermal conditions for 11 hours at the temperature of interest.

Fourier-Transform Infrared spectroscopy (FT-IR) measurements were carried out on a Thermo Scientific Nicolet IR100 FT-IR in a nitrogen filled glovebox over the range of 500 to $3500 \mathrm{~cm}^{-1}$. Samples were prepared as $\mathrm{KBr}$ pellets with a sample to $\mathrm{KBr}$ weight ratio of approximately 1:100. X-ray photoelectron measurements were performed using a PHI 3056 XPS spectrometer utilizing an Al X-ray source operated at 350W and $15 \mathrm{kV}$. Samples were pressed into indium sheets mounted on a sample holder in an argon filled glove box. The samples were load locked from the glove box into the XPS to prevent surface oxidation in a specially built transfer device. Charge calibrations, on the order of $0.2 \mathrm{eV}$, were made by assigning the first $\mathrm{C}$ peak a value of $284.8 \mathrm{eV}$. Hydrogen physisorption measurements were carried out on a Quantachrome AUTOSORB-1C instrument (Quantachrome Corporation, USA) using research grade hydrogen ( $99.9999 \%$ purity). Measurements were made at $77 \mathrm{~K}$ with 40 adsorption points and 40 desorption points in a $9 \mathrm{~mm}$ cell with rod insert. Samples of approximately $100 \mathrm{mg}$ were degassed for 1 hour at $300{ }^{\circ} \mathrm{C}$ prior to measurements. Hydrogen adsorption isotherms were fit to the one-binding site Langmuir model. 


\section{Results and Discussion}

\subsection{Physisorption}

A series of physisorption measurements demonstrate that alkali metal doping enhances the hydrogen physisorption capability of fullerenes. Increased hydrogen uptake was noticed in fullerenes doped with alkali metals via both methods. Although the crystalline nature of $\mathrm{C}_{60}$ limits the accessible surface area resulting in low hydrogen capacities at ambient pressures, the focus of the study is to evaluate the effect of the dopant on the physisorption interaction of hydrogen with $\mathrm{C}_{60}$. Variable temperature isotherms of $\mathrm{Li}_{12} \mathrm{C}_{60}$ (Figure $1 \mathrm{~A}$ ) and pure $\mathrm{C}_{60}$ (Supporting Information) were used to calculate the isosteric heats of adsorption of the materials. $\left(2.97 \mathrm{KJ} / \mathrm{mol}\right.$ for $\mathrm{Li}_{12} \mathrm{C}_{60}$ and $0.486 \mathrm{KJ} / \mathrm{mol}$ for $\mathrm{C}_{60}$ ) These measurements demonstrate that alkali metal doping of fullerenes increase the isosteric heats of adsorption (Figure 1B) which improve the hydrogen uptake compared to pure $\mathrm{C}_{60}$. Figure 2 shows the enhancement in hydrogen uptake via physisorption for the $\mathrm{Li}$ and Na hydride doped $\mathrm{C}_{60}\left(\mathrm{M}_{6} \mathrm{C}_{60}\right)$ compared to pure $\mathrm{C}_{60}$ at $77 \mathrm{~K}$. Hydrogen uptake was increased more than an order of magnitude for the hydride doped fullerenes $\mathrm{Na}_{6} \mathrm{C}_{60}$ and $\mathrm{Li}_{6} \mathrm{C}_{60}$. At $1 \mathrm{~atm}$ the hydrogen uptake was $14.69 \mathrm{cc} / \mathrm{gram}, 8.64 \mathrm{cc} / \mathrm{gram}$, and $0.39 \mathrm{cc} /$ gram for the $\mathrm{Li}_{6} \mathrm{C}_{60}, \mathrm{Na}_{6} \mathrm{C}_{60}$, and pure $\mathrm{C}_{60}$ respectively. 
A)

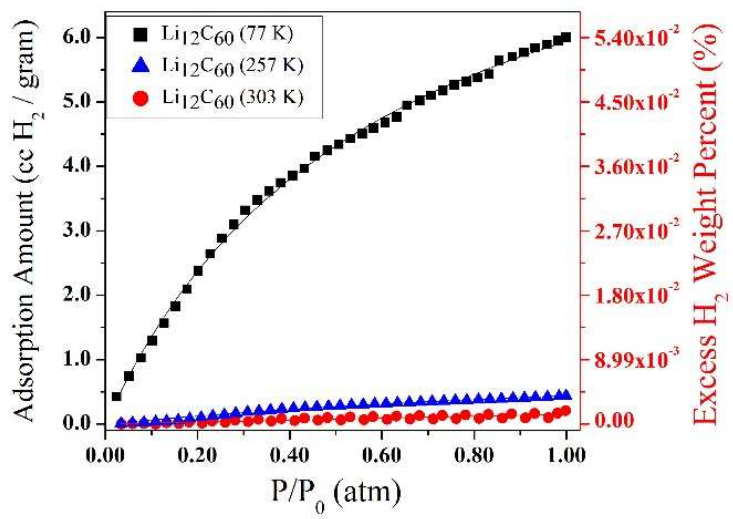

B)

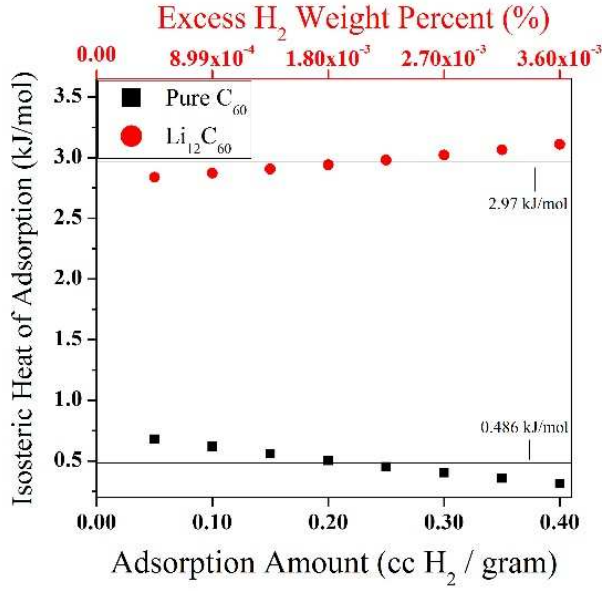

Figure 1: A) Hydrogen isotherms of $\mathrm{Li}_{12} \mathrm{C}_{60}$ at different temperatures B) Isosteric heats of adsorption for $\mathrm{Li}_{12} \mathrm{C}_{60}$ and $\mathrm{C}_{60}$ for an adsorption amount up to $0.40 \mathrm{cc} / \mathrm{gram}$

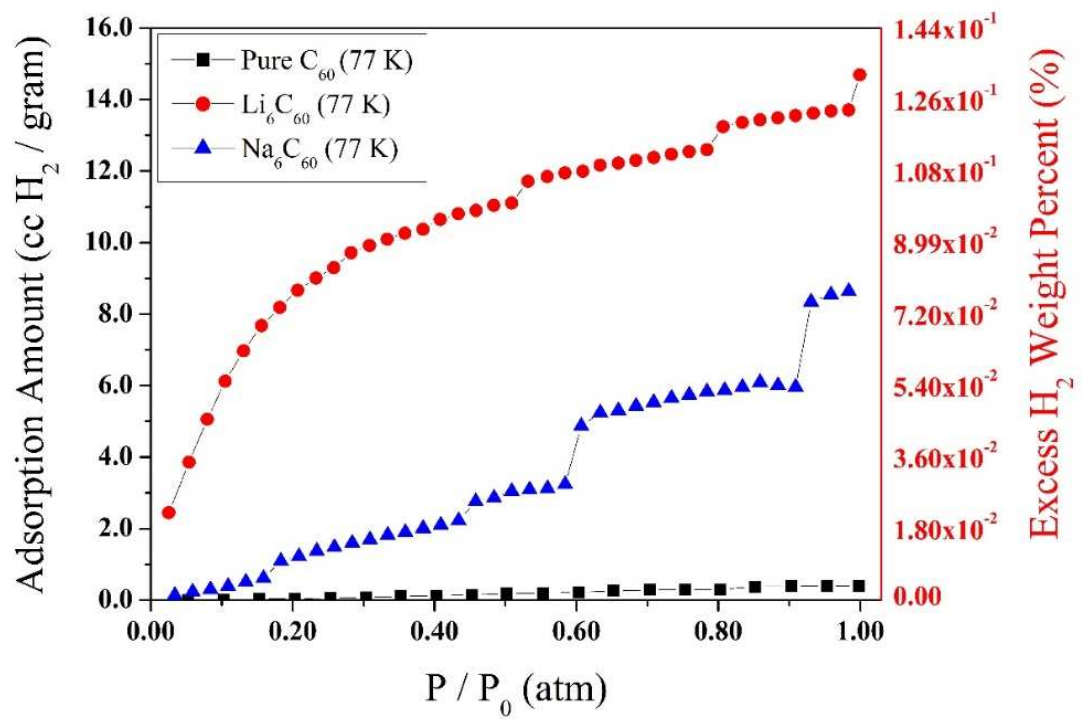

Figure 2: Hydrogen physisorption curves at $77 \mathrm{~K}$ for $\mathrm{C}_{60}$ (black squares), $\mathrm{Na}_{6} \mathrm{C}_{60}$ (blue triangles), and $\mathrm{Li}_{6} \mathrm{C}_{60}$ (red circles) from hydride synthesis. 


\subsection{Chemisorption}

The chemisorption properties of alkali doped fullerenes were further investigated to determine the possibilities of forming highly hydrogenated fullerene species $\left(>\mathrm{C}_{60} \mathrm{H}_{48}\right)$. Figure 3 shows the positive mode mass spectra of hydrogenated $\mathrm{Na}_{6} \mathrm{C}_{60}$ and $\mathrm{Li}_{6} \mathrm{C}_{60}$ synthesized via liquid ammonia. Evidence for hydrogenated fullerenes up to $\mathrm{C}_{60} \mathrm{H}_{36}$ is clearly apparent in both lithium and sodium doped fullerenes hydrogenated at $300{ }^{\circ} \mathrm{C}$ and 100 bar of hydrogen overpressure. Laser desorption ionization time-of-flight mass spectrometry (LDI-TOF-MS) measurements confirm higher levels of hydrogenation for the alkali doped fullerenes than for pure $\mathrm{C}_{60}$ which displays a mass distribution corresponding to less than $\mathrm{C}_{60} \mathrm{H}_{18}$. (Supporting Information) The alteration of intensities between even and odd hydrogen containing ions (every $1 \mathrm{amu}$ ) is characteristic of LDI-TOF-MS spectra of hydrogenated fullerenes as shown in many works. ${ }^{[13,16,18]}$ Higher levels of hydrogenation in alkali doped fullerenes via the hydride doped synthetic methods have been demonstrated in our previous work. ${ }^{[16,18]}$ In this case, we demonstrate that alkali metal doped fullerenes created via the liquid ammonia synthesis also facilitate the chemisorption of hydrogen onto the fullerene cage. 

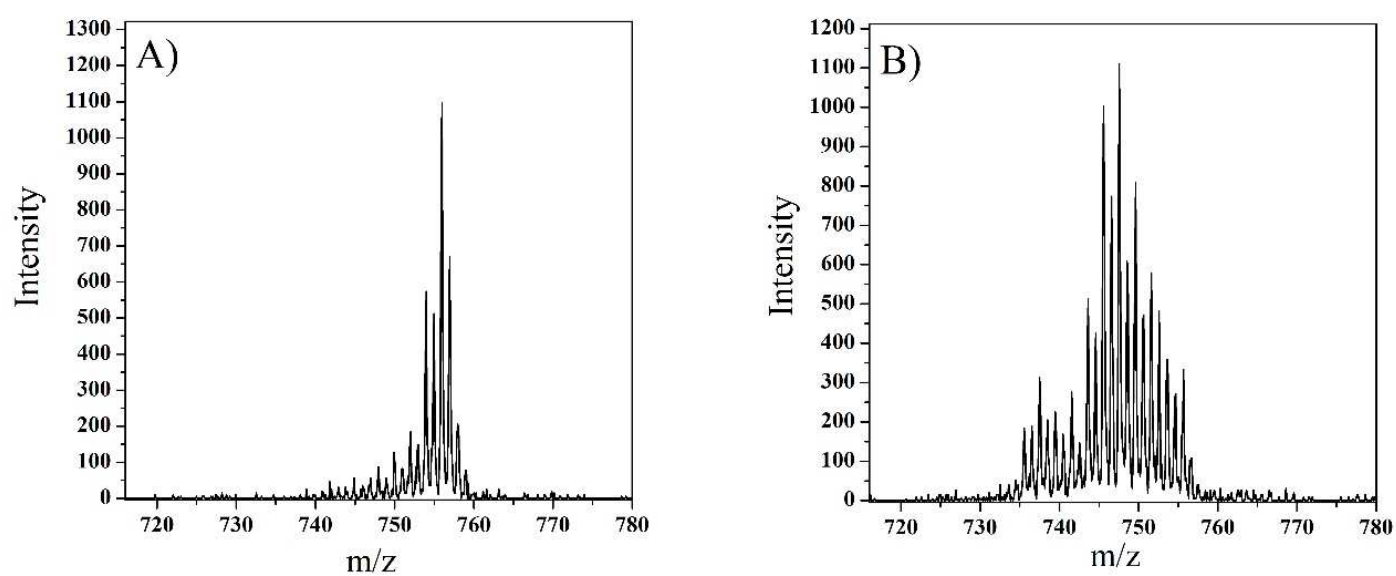

Figure 3: Positive mode LDI-TOF-MS spectra of alkali doped fullerenes synthesized via liquid ammonia and hydrogenated at $300^{\circ} \mathrm{C}$ and 100 bar $\mathrm{H}_{2}$. A) $\mathrm{C}_{60} \mathrm{H}_{\mathrm{x}}$ from $\mathrm{Na}_{6} \mathrm{C}_{60}$ B) $\mathrm{C} 60 \mathrm{Hx}$ from $\mathrm{Li}_{6} \mathrm{C}_{60}$

FT-IR was utilized to show the similarity between the $\mathrm{Na}$ and $\mathrm{Li}$ doped $\mathrm{C}_{60}$ synthesized by alkali intercalation using liquid ammonia. The spectra displayed in Figure 4 shows the characteristic $\mathrm{F}_{1 \mathrm{u}}$ vibrational modes of $\mathrm{C}_{60}\left(527 \mathrm{~cm}^{-1}, 577 \mathrm{~cm}^{-1}, 1183 \mathrm{~cm}^{-1}, 1428 \mathrm{~cm}^{-1}\right)$ in the alkali doped fullerenes synthesized in liquid ammonia. ${ }^{[20]}$ Broad peaks at $\sim 2845 \mathrm{~cm}^{-1}$ and $\sim 2914$ $\mathrm{cm}^{-1}$, representing $\mathrm{sp}^{3}$ hybridized $\mathrm{C}-\mathrm{H}$ stretching modes, are observed for the alkali doped fullerenes that were hydrogenated at $300{ }^{\circ} \mathrm{C}$ and 100 bar hydrogen. The disappearance of the $\mathrm{F}_{1 \mathrm{u}}$ vibrational modes in the hydrogenated samples is consistent with a breaking of the symmetry from hydrogen addition which is consistent with previous studies. ${ }^{[21]}$ 


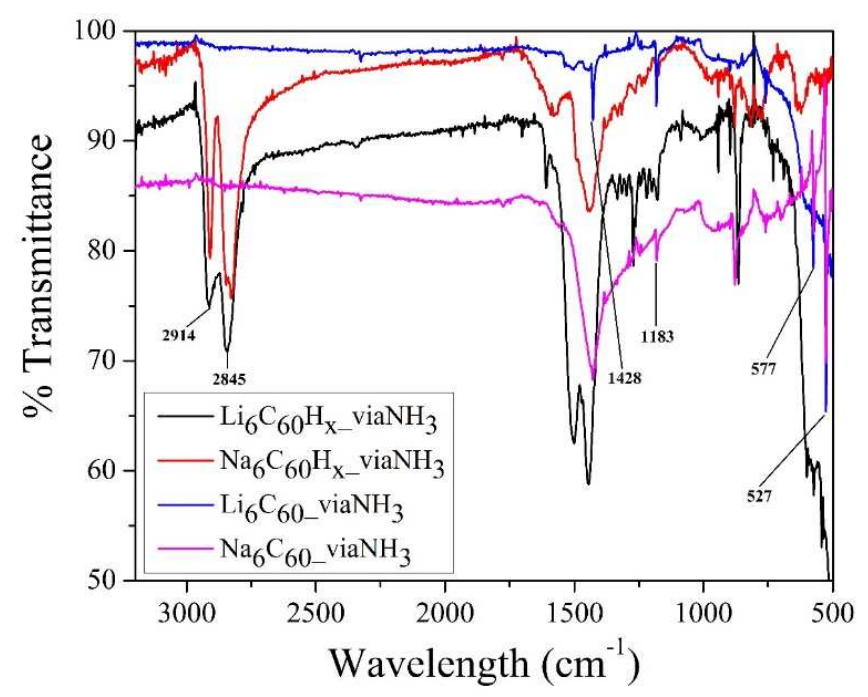

Figure 4: FT-IR spectra of Li and Na doped fullerenes synthesized via liquid ammonia in the hydrogenated and as prepared state.

To further understand the surface chemistry of the materials $\mathrm{Na}$ 1s and Li 1s XPS spectra were collected for the samples (presented in Figure 5) along with appropriate reference compounds. For the lithium doped fullerenes, there is a clear $2 \mathrm{eV}$ shift in the Li species in the hydride doped samples to higher binding energies compared to the $\mathrm{NH}_{3}$ mixed samples. This is consistent with a slight oxidation of $\mathrm{Li}$ or a decrease in electron density on the $\mathrm{Li}$. The lower $\mathrm{Li}$ binding energy measured for pure $\mathrm{LiH}$ suggests that there is more electron density donation to the fullerene than to the hydride indicating the Li bound to the surface is more cationic in nature. A similar $1 \mathrm{eV}$ shift in binding energy is observed between the hydride doped and $\mathrm{NH}_{3}$ mixed sodium intercalated fullerenes. The re(de)hydrogenated samples (produced by the hydride doped synthesis) show similar binding energies as the starting material $\left(\mathrm{MH}: \mathrm{C}_{60}\right)$ indicating that the alkali metals are present in similarly oxidized conditions. 

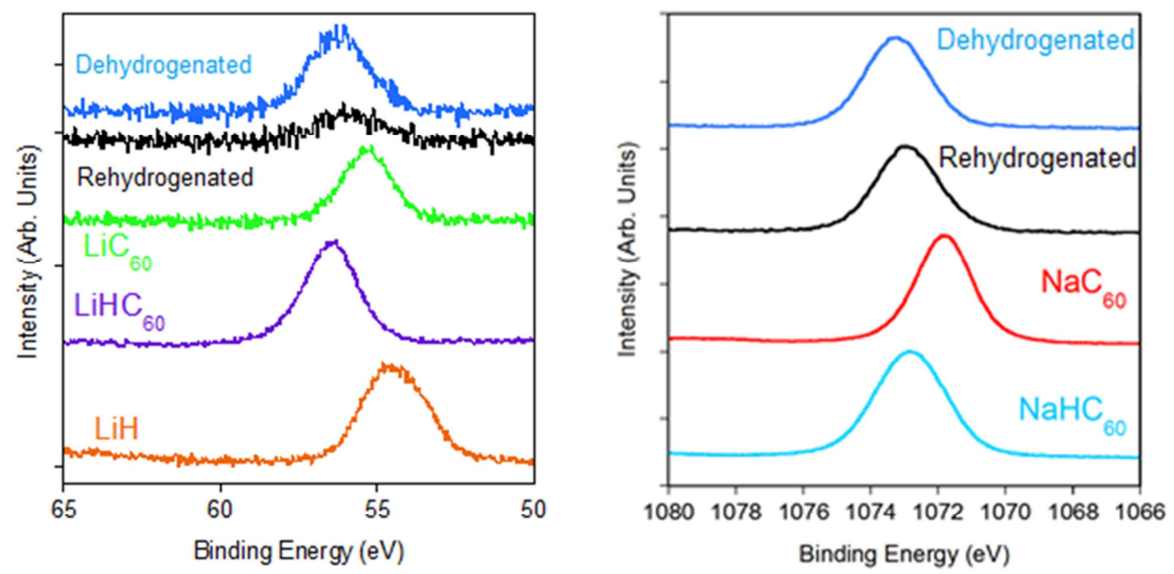

Figure 5: XPS spectra for the $\mathrm{Na}$ and Li doped fullerenes synthesized by the hydride doping method (MH: $\left.\mathrm{C}_{60}\right)$ and the liquid ammonia method $\left(\mathrm{M}: \mathrm{C}_{60}\right)$. The re(de)hydrogenated species are the hydride doped samples.

In our continued effort to characterize the formation of alkali doped fullerenes under higher temperatures and pressures, we turned our focus to the chemisorption of hydrogen in $\mathrm{Na}_{6} \mathrm{C}_{60}$. The degree of hydrogenation in the material as a function of pressure and temperature was monitored by LDI-TOF-MS. A series of mass peaks consistent with the formation of $\mathrm{C}_{60} \mathrm{H}_{60}$ was observed when the sample was hydrogenated at $350^{\circ} \mathrm{C}$ and 100 bar $\mathrm{H}_{2}$. Figure 6 shows the mass spectra of sodium doped fullerenes, synthesized by hydride doping and hydrogenated at $350{ }^{\circ} \mathrm{C}$. The spectra display a large intensity distribution corresponding to $\mathrm{C}_{60} \mathrm{H}_{36}$, as evidenced by the peak ending at $755 \mathrm{~m} / \mathrm{z}\left(\mathrm{C}_{60} \mathrm{H}_{35}{ }^{+}\right)$, with another distribution consistent with $\mathrm{C}_{60} \mathrm{H}_{60}$ at 780 $\mathrm{m} / \mathrm{z}\left(\mathrm{C}_{60} \mathrm{H}_{60}{ }^{+}\right)$. In order to confirm the identity of the higher mass distribution, a series of mass spectrometric experiments were carried out to rule out other possible ions. First, the material was exposed to air overnight to insure that the distribution was not the result of oxidation. Figure 6B displays a mass distribution for the oxidized material shifted up by $16 \mathrm{~m} / \mathrm{z}$ with no significant changes in intensity for the other distributions. $\mathrm{Na}_{6} \mathrm{C}_{60}$ was then deuterated in order to observe a shift by 2 amu for each deuterium compared to the $1 \mathrm{~m} / \mathrm{z}$ shift for each hydrogen. The mass distributions observed for the deuterated fulleranes shifted by $36 \mathrm{~m} / \mathrm{z}$ for the first distribution and 
by $60 \mathrm{~m} / \mathrm{z}$ for the second distribution which is consistent with $\mathrm{C}_{60} \mathrm{D}_{36}$ and $\mathrm{C}_{60} \mathrm{D}_{60}$ respectively. For comparison purposes, $\mathrm{Na}_{6} \mathrm{C}_{60}$ was deuterated at $290{ }^{\circ} \mathrm{C}$ and only displays a mass distribution corresponding to $\mathrm{C}_{60} \mathrm{D}_{36}(792 \mathrm{~m} / \mathrm{z})$ demonstrating that the higher hydrogenation temperature favors the formation of $\mathrm{C}_{60} \mathrm{D}_{60}$. It is highly unlikely that this higher mass distribution is due to a sodium atom attached to the cage since this is not observed in the lower temperature hydrogenated samples. Furthermore, this would mean that deuterated samples would only attach 2 sodium atoms and hydrogenated samples would only attach 1 sodium atom. Again this is improbable and no higher mass distributions corresponding to multiple sodium atom addition is observed. Although there have been previous reports of $\mathrm{C}_{60} \mathrm{H}_{60},{ }^{[22-24]}$ to our knowledge this LDITOF-MS data is the first to show mass spectrometric evidence for the existence of $\mathrm{C}_{60} \mathrm{H}_{60}$. Previous reports for the production of $\mathrm{C}_{60} \mathrm{H}_{60}$ utilized gravimetric methods to show that the weight change of the material corresponded to 60 hydrogens per fullerenes or isothermal conditions showing a pressure drop of hydrogen that corresponded to 60 hydrogens per fullerene cage. Meletov et al. used combustion analysis to confirm the hydrogen content in their material and Raman spectroscopy to show an increase in symmetry of the material as evidenced by a decrease in the number of vibrational modes. Although these techniques demonstrate hydrogen capacities corresponding to $\mathrm{C}_{60} \mathrm{H}_{60}$, none of these techniques completely rule out the possibility of cage fragmentation into polyaromatic hydrocarbons. This is known to occur in pure $\mathrm{C}_{60}$ hydrogenated under extreme conditions. ${ }^{[25]}$ 

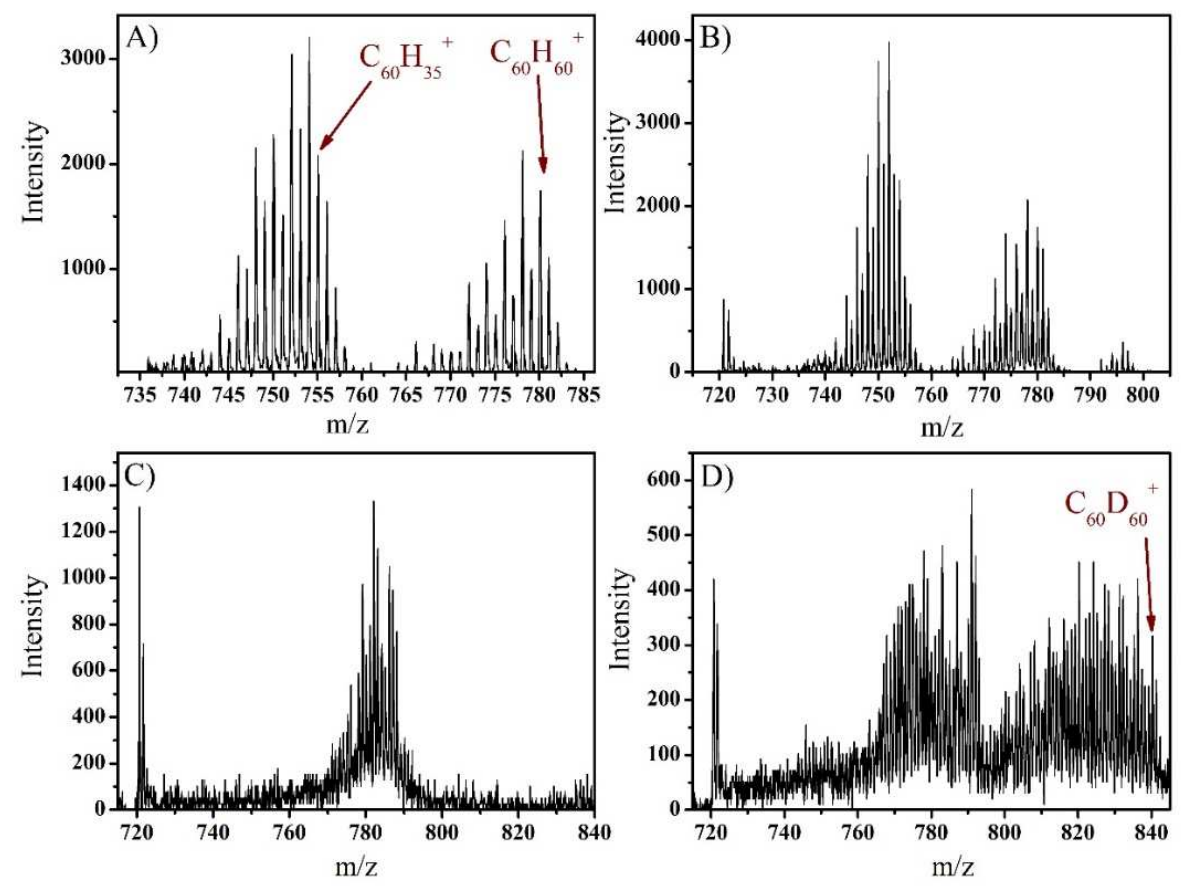

Figure 6: Positive mode LDI-TOF-MS spectra of hydride doped $\mathrm{Na}_{6} \mathrm{C}_{60} \mathrm{~A}$ ) hydrogenated at $350{ }^{\circ} \mathrm{C}$ and 100 bar $\mathrm{H}_{2}$

B) hydrogenated at $350{ }^{\circ} \mathrm{C}$ and 100 bar $\mathrm{H}_{2}$ air exposed overnight C) deuterated at $290{ }^{\circ} \mathrm{C}$ and 100 bar $\mathrm{D}_{2} \mathrm{D}$ ) deuterated at $350{ }^{\circ} \mathrm{C}$ and 100 bar $\mathrm{D}_{2}$

\section{Conclusion}

The physisorption measurements display low hydrogen uptake in the alkali doped $\mathrm{C}_{60}$ samples under $1 \mathrm{~atm} \mathrm{H}_{2}$ and $77 \mathrm{~K}$. However, the alkali doped samples demonstrated an order of magnitude increase in the hydrogen uptake relative to the pure $\mathrm{C}_{60}$ sample. We also observed an increase in the isosteric heat of absorption $(2.97 \mathrm{~kJ} / \mathrm{mol})$ compared to pure $\mathrm{C}_{60}(0.486 \mathrm{~kJ} / \mathrm{mol})$. These findings demonstrate that the interaction strength between hydrogen and $\mathrm{C}_{60}$ is increased upon alkali doping with both sodium and lithium. The enhancement of adsorption upon alkali doping is believed to be due to the differential charge gradient produced on the surface of the 
fullerene which gives rise to enhanced dipole interactions. The low hydrogen adsorption capacity is attributed to the low BET surface areas for $\mathrm{C}_{60}$. Previous studies using nitrogen for BET experiments presented an average surface area less than $1 \mathrm{~m}^{2} / \mathrm{g} .{ }^{[26]}$ This is virtually negligible compared to the surface areas of MOF's which typically have surface areas greater than 2000 $\mathrm{m}^{2} / \mathrm{g}$. ${ }^{[27]}$ The low surface area of $\mathrm{C}_{60}$ is attributed to the highly crystalline state and small pore sizes of our materials. To overcome this problem, recent theoretical work proposed the possibility of incorporating Li doped $\mathrm{C}_{60}$ into a porous aromatic framework (PAF) with the consequent enhancement of the composite's physisorption capacity. ${ }^{[28]}$ It has also been reported that hydrogen uptake can be enhanced in pure $\mathrm{C}_{60}$ by high temperature activation with oxygen or carbon dioxide. ${ }^{[29,30]}$ These enhancements are likely due to an increased accessible surface area upon cage alteration. On the other hand, enhancements shown in our materials are due to the dopant $(\mathrm{Na}, \mathrm{Li})$ present in our materials and not an increase in accessible surface area.

Alkali doped fullerenes synthesized by the different techniques demonstrated in this work display slight differences in the hydrogen storage capacities via either a chemisorption or a physisorption mode. The differences observed are likely due to variable localized alkali metal clustering or changes in the alkali metal's locations on the fullerene due to the synthetic method used. Alkali doping also enhanced the chemisorption properties of fullerenes resulting in higher degrees of hydrogenation compared to pure $\mathrm{C}_{60}$. It is evident that alkali doping of $\mathrm{C}_{60}$ alters the electronic properties of $\mathrm{C}_{60}$ as it enhances the interaction with hydrogen. Therefore, the most logical hypothesis is that the alkali metal is either dispersed differently within the two materials or has differing bonding with the fullerene cage. This may be due to a difference in the position on the fullerene cage that the alkali metal occupies or a difference in the number of alkali atoms on each fullerene cage. Although the molar ratio of alkali metal to $\mathrm{C}_{60}$ in each case is 
predetermined, the most stable and preferred number of alkali metal atoms on each fullerene cage may be different for each synthetic technique. Alkali decorated fullerenes synthesized by both techniques (hydride doped and $\mathrm{NH}_{3}$ mixed) show enhancements in the chemisorption and physisorption properties of hydrogen compared to pure $\mathrm{C}_{60}$. The enhancement in both physisorption and chemisorption properties is most likely due to the nature of the charge differential created from the electron density donation to the fullerene cage from the alkali metal.

We also present the first spectroscopic evidence for the formation of $\mathrm{C}_{60} \mathrm{H}_{60}$ when the material is doped with sodium. To our knowledge this is the first reported mass spectrometric confirmation of the molecular species $\mathrm{C}_{60} \mathrm{H}_{60}$. Deuteration experiments confirm that the additional mass observed on the $\mathrm{C}_{60}$ is from additional hydrogen and not another element. Currently the $\mathrm{C}_{60} \mathrm{H}_{60}$ is present in a mixture of other hydrogenated fullerenes, but these finding indicate that it may be possible to isolate $\mathrm{C}_{60} \mathrm{H}_{60}$ in pure form. Attempts to isolate and characterize $\mathrm{C}_{60} \mathrm{H}_{60}$ and other highly hydrogenated fullerene species $\left(>\mathrm{C}_{60} \mathrm{H}_{48}\right)$ are currently under investigation.

\section{Acknowledgments}

This research was supported by National Science Foundation Grant DGE0801470, "Sustainable Technology through Advanced Interdisciplinary Research" (STAIR), awarded to the University of Tennessee Knoxville. Work at SRNL was supported by the U.S. Department of Energy, Office of Science, Basic Energy Sciences, Materials Sciences and Engineering Division. XPS studies (GMV) were supported by the U.S. Department of Energy, Office of Science, Basic Energy Sciences, Materials Sciences and Engineering Division. A portion of this research was conducted at the Center for Nanophase Materials Sciences, which is sponsored at Oak Ridge 
National Laboratory by the Scientific User Facilities Division, Office of Basic Energy Sciences, U.S. Department of Energy.

\section{References}

1) Jena, P. Materials for Hydrogen Storage: Past, Present, and Future. J. Phys. Chem. Letters, 2011, 2, 206-211.

2) Chandrakumar, K. R. S.; Srinivasu, K.; Ghosh, S. K. Nanoscale Curvature-Induced Hydrogen Adsorption in Alkali Metal Doped Carbon Nanomaterials. J. Phys. Chem. C 2008, $112,15670-15679$.

3) Sun, Q.; Jena, P.; Wang, Q.; Marquez, M. First-Principles Study of Hydrogen Storage on $\mathrm{Li}_{12} \mathrm{C}_{60} . J$. Am. Chem. Soc. 2006, 128, 9741-9745.

4) Chandrakumar, K. R. S.; Ghosh, S. K. Alkali-Metal-Induced Enhancement of Hydrogen Adsorption in $\mathrm{C}_{60}$ Fullerene: An Ab Initio Study. Nano Letters 2008, 8 (1), 13-19.

5) Jensen, C. M.; Sun, D.; Raman, S. S.; Murphy, K.; Niemczura, W. P.; Kumashiro, K. K.; et al. Catalytically enhanced systems for hydrogen storage. Proceedings for the 2002 U.S. DOE Hydrogen Program Review.

6) Shane, D. T.; Corey, R.; Rayhel, L.; Wellons, M.; Teprovich, J. A.; Zidan, R.; Hwang, S.; Bowman, R.; Conradi, M. S. NMR Study of $\mathrm{LiBH}_{4}$ with $\mathrm{C}_{60}$. J. Phys. Chem. C 2010, $114,19862-19866$.

7) Teprovich, J. A.; Knight, D.; Wellons, M.; Zidan, R. Catalytic Effect of Fullerene and Formation of Nanocomposites with Complex Hydrides. J. Alloys Compd. 2011, 509, S562-S566. 
8) Ward, P. A.; Teprovich, J. A.; Peters, B.; Wheeler, J.; Compton, R. N.; Zidan, R.

Reversible Hydrogen Storage in a $\mathrm{LiBH}_{4}-\mathrm{C}_{60}$ Nanocomposite. J. Phys. Chem. C 2013, $117,22569-22575$.

9) Berseth, P. A.; Harter, A. G.; Zidan, R.; Blomqvist, A.; Araujo, C. M.; Scheicher, R. H.; Ahuja, R.; Jena, P. Carbon Nanomaterials as Catalysts for Hydrogen Uptake and Release in $\mathrm{NaAlH}_{4}$. Nano Letters 2009, 9 (4), 1501-1505.

10) Paolone, A.; Vico, F.; Teocoli, F.; Sanna, S.; Palumbo, O.; Cantelli, R.; Knight, D.; Teprovich, J. A.; Zidan, R. Relaxation Processes and Structural Changes in Li and $\mathrm{Na}$ Doped Fulleranes for Hydrogen Storage. J. Phys. Chem. C 2012, 116, 16365-16370.

11) Paolone, A.; Palumbo, O.; Leardini, F.; Cantelli, R.; Knight, D.; Teprovich, J. A.; Zidan, R. A Spectroscopic Investigation of Hydrogenated Li Doped Fullerene. J. Alloys Compd. 2013, 580, S67-S69.

12) Teprovich, J. A. Jr.; Knight, D. A.; Peters, B.; Zidan, R. Comparative study of reversible hydrogen storage in alkali-doped fulleranes. J. Alloys Compd. 2013, 580, S364-S367.

13) Jin, C.; Hettich, R.; Compton, R.; Joyce, D.; Blencoe, J.; Burch,T. Direct Solid-Phase Hydrogenation of Fullerenes. J. Phys. Chem. 1994, 98, 4215-4217.

14) Talyzin, A. V.; Tsybin, Y. O.; Purcell, J. M.; Schaub, T. M.; Shulg, Y. M.; Noreus, D.; et al. Reaction of Hydrogen Gas with $\mathrm{C}_{60}$ at Elevated Pressure and Temperature: Hydrogenation and Cage Fragmentation. J. Phys. Chem. A 2006, 110, 8528-8534.

15) Luzan, S. M.; Tsybin, Y. O.; Talyzin, A. V. Reaction of $\mathrm{C}_{60}$ with Hydrogen Gas: In Situ Monitoring and Pathways. J. Chem. Phys. C 2011, 115, 11484-11492. 
16) Teprovich, J. A.; Wellons, M. S.; Lascola, R.; Hwang, S.; Ward, P. A.; Compton, R. N.; et al. Synthesis and Characterization of a Lithium-Doped Fullerane $\left(\mathrm{Li}_{\mathrm{x}}-\mathrm{C}_{60}-\mathrm{H}_{\mathrm{y}}\right)$ for Reversible Hydrogen Storage. Nano Lett. 2012, 12, 582-589.

17) Mauron, P.; Gaboardi, M.; Remhof, A.; Bliersbach, A.; Sheptyakov, D.; Aramini, M.; Vlahopoulou, G.; Giglio, F.; Pontiroli, D.; Ricco, M.; Zuttel, A. Hydrogen Sorption in $\mathrm{Li}_{12} \mathrm{C}_{60}$. J. Phys. Chem. C 2013, 117, 22598-22602.

18) Knight, D. A.; Teprovich, J. A. Jr.; Summers, A.; Peters, B.; Ward, P. A.; Compton, R. N.; Zidan, R. Synthesis, characterization, and reversible hydrogen sorption study of sodium-doped fullerene. Nanotechnology 2013, 24, 455601 (7pgs).

19) Mauron, P.; Remhof, A.; Bliersbach, A.; Borgschulte, A.; Zuttel, A.; Sheptyakov, D.; Gaboardi, M.; Choucair, M.; Pontiroli, D.; Aramini, M.; Gorreri, A.; Ricco, M. Reversible hydrogen absorption in sodium intercalated fullerenes. Int. J. Hydrogen Energy 2012, 37, 14307-14314.

20) Rao, A. M.; Eklund, P. C.; Hodeau, J.-L.; Marques, L.; Nunez-Reguerio, M. Phys. Rev. B $1997,55,4766-4773$

21) Bini, R.; Ebenhoch, J.; Fanti, M.; Fowler, P. W.; Leach, S.; Orlandi, G.; Ruchardt, Ch.; Sandall, J. P. B.; Zerbetto, F. The vibrational spectroscopy of $\mathrm{C}_{60} \mathrm{H}_{36}$ : An experimental and theoretical study. Chem. Phys. 1998, 232, 75-94.

22) Cataldo, F.; Milani, P. Fulleranes: The Hydrogenated Fullerenes, Springer, 2010, New York, NY.

23) Meletov, K. P.; Bashkin, I. O.; Shestakov, V. V.; Tartakovskii, I. I.; Maksimov, A. A.; Arvanitidis, J.; Christofilos, D.; Kourouklis, G. A. Comparative Raman Study of the 
$\mathrm{C}_{60} \mathrm{H}_{36}$ and $\mathrm{C}_{60} \mathrm{H}_{60}$ Fullerene Hydrides. Fullerenes, Nanotubes, and Carbon Nanostructures, 2008, 16, 593-596.

24) Schur, D. V.; Zaginaichenko, S. Y.; Savenko, A. F.; Bogolepov, V. A.; Anikina, N. S.; Zolotarenko, A. D.; Matysina, Z. A.; Veziroglu, T. N.; Skryabina, N. E. Experimental evaluation of total hydrogen capacity for fullerite $\mathrm{C}_{60}$. Int. J. Hydrogen Energy 2011, 36, $1143-1151$.

25) Talyzin, A. V.; Luzan, S.; Anoshkin, I. V.; Nasibulin, A. G.; Kauppinnen, E. I.;

Dzwilewski, A.; Kreta, A.; Jamnik, J.; Hassanien, A.; Lundstedt, A.; Grennberg, H. Hydrogen-Driven Cage Unzipping of $\mathrm{C}_{60}$ into Nano-Graphenes. J. Phys. Chem. C 2014, $118(12), 6504-6513$.

26) Mackeyev, Y. A.; Marks, J. W.; Rosenblum, M. G.; Wilson, L. J. Stable Containment of Radionuclides on the Nanoscale by Cut Single-Wall Carbon Nanotubes. J. Phys. Chem. B 2005, 109, 5482-5484.

27) Furukawa, H.; Cordova, K. E.; O’Keeffe, M.; Yaghi, O. M. The Chemistry and Applications of Metal-Organic Frameworks. Science 2013, 341, 1230444.

28) Ahmed, A.; Thornton, A. W.; Konstas, K. Kannam, S. K.; Babarao, R.; Todd, B. D.; Hill, A. J.; Hill, M. R. Strategies toward Enhanced Low-Pressure Volumetric Hydrogen Storage in Nanoporous Cryoadsorbents. Langmuir 2013, 29 (50), 15689-15697.

29) Deng, S.; Saha, D. Hydrogen adsorption on partially truncated and open cage C60 fullerene. Carbon 2010, 48, 3471-3476.

30) Loutfy, R. O.; Wexler, E. M. Feasibility of Fullerene Hydride as A High Capacity Hydrogen Storage Material. Proceedings of the 2001 DOE Hydrogen Program Review, 2001. 